\title{
PRODUÇÃo DE MUDAS DE RABANETE EM DIFERENTES SUBSTRATOS ORGÂNICOS
}

\author{
Israel Martins Pereira ${ }^{1}$ \\ Rebyson Bissaco Guidinelle ${ }^{2}$ \\ Alex Justno Jacarias ${ }^{3}$ \\ Júlio Cesar Gradice Saluci ${ }^{4}$ \\ Mário Euclides Pechara da Costa Jaeggi ${ }^{5}$ \\ Ronald Martins Pereira Junior ${ }^{6}$ \\ Maxwel Rodrigues Nascimento ${ }^{7}$ \\ Rogério Rodrigues Rangel ${ }^{8}$ \\ Jhonatas Souza Ventura Tosta ${ }^{9}$ \\ Wallace Luís de Lima $^{10}$
}

Resumo: O objetivo deste trabalho foi avaliar diferentes tipos de substratos na produção de plântulas de rabanete com sementes provenientes de envelope aluminizados e latas. $O$ delineamento experimental utilizado foi o inteiramente casualizado disposto no esquema fatorial 4x2, sendo 4 substratos: $S 1$ - Esterco bovino curtido; S2 - Comercial; S3 - Vermicomposto e S4 - Composto orgânico de podas de jardim e esterco bovino (\%), e sementes de 2 embalagens comerciais sendo envelope aluminizado e a lata. Os substratos comercial, vermicomposto e composto a base esterco e poda de jardins, proporcionaram os melhores desenvolvimentos para todos os parâmetros morfoagronômicos estudados nas plântulas de rabanete. As sementes provenientes embalagem de lata promoveram os melhores desenvolvimentos para todos os parâmetros estudados nos substratos testados.

Palavras-chave: Raphanus sativus L; Resíduo orgânico; Produção de mudas.

\footnotetext{
1 Tecnologia em Cafeicultura/Instituto Federal do Espirito Santo - Campus de Alegre, Brasil. E-mail: israelmartins80@gmail.com.

2 Tecnologia em Cafeicultura/Instituto Federal do Espirito Santo - Campus de Alegre, Brasil. E-mail: rebysonguidinelle@gmail.com.

3 Tecnologia em Cafeicultura/Instituto Federal do Espirito Santo - Campus de Alegre, Brasil. E-mail: alexjustino12@gmail.com.

4 Tecnologia em Cafeicultura/Instituto Federal do Espirito Santo - Campus de Alegre, Brasil. E-mail: juliosaluci@gmail.com.

5 Produção Vegetal/Universidade Estadual Norte Fluminense Darcy Ribeiro, Centro de Ciências e Tecnologias Agropecuárias, Brasil. E-mail: mariopechara@hotmail.com.

6 Engenharia Agronômica/Universidade Federal do Espírito Santo, Centro de Ciências Agrárias - Campus de Alegre, Brasil. E-mail: ronal_martins_pereira@hotmail.com.

7 Produção Vegetal/Universidade Estadual Norte Fluminense Darcy Ribeiro, Centro de Ciências e Tecnologias Agropecuárias, Brasil. E-mail: maxwel.m88@gmail.com.

${ }^{8}$ Engenharia Agrícola/Universidade Federal de Lavras, Brasil. E-mail: rogeriorr7@hotmail.com.

9 Tecnologia em Cafeicultura/Instituto Federal do Espirito Santo - Campus de Alegre, Brasil. E-mail: jhonatastosta@hotmail.com.

10 Professor D.Sc. Agronomia - Ciência do Solo no Instituto Federal do Espirito Santo - Campus de Alegre, Brasil. E-mail: wallace@ifes.edu.br.
} 